Z Herz- Thorax- Gefäßchir 2019 · 33:224-225 https://doi.org/10.1007/s00398-019-0313-7 Online publiziert: 30. April 2019

(c) Springer Medizin Verlag GmbH, ein Teil von Springer Nature 2019

Es wird schwer sein, auch nur eine historische Darstellung über die Entwicklung der klinischen Kardiologie zu finden, in der nicht Helen Taussig (1898-1986) als die Begründerin und die Galionsfigur der Kardiologie angeborener Fehlbildungen des Herzens dargestellt sein wird (• Abb. 1).

Am 24. Mai 1898 wurde Helen Brooke Taussig in Cambridge, MA, USA, als viertes Kind einer angesehenen amerikanischen Ostküsten-Familie geboren. Bereits früh musste sie ihren lebenslangen Kampf um ihre persönliche und später professionelle Entwicklung aufnehmen. Mit 11 Jahren verlor sie ihre Mutter durch eine Tuberkulose. Eine schwere angeborene Lese- und Rechtschreibschwäche, die sie bis in ihre späten Jahre nicht ganz überwinden konnte, erschwerte ihre schulische Entwicklung erheblich. Dennoch konnte sie 1921 ihre Studien, zunächst am Radcliffe College in Cambridge, MA, anschließend an der Universität Berkeley, CA, nicht nur mit guten Leistungen und einem BA abschließen, sondern auch mit der Universitäts-Championship im Tennis. Ihren Wunsch, anschließend Medizin $\mathrm{zu}$ studieren, konnte sie jedoch nicht verwirklichen, da dieser Studiengang zu diesem Zeitpunkt noch ausschließlich Männern vorbehalten war. So widmete sie sich zunächst einer extracurriculären Ausbildung in Anatomie und Histologie. Als Sektionsmaterial wurde ihr dazu ein Rinderherz zum Studium von Muskelbündeln vorgelegt - ihr Einstieg in die Kardiologie.

Erst ein Wechsel an die Johns Hopkins School of Medicine in Baltimore, MD, 1923 gab ihr die Gelegenheit, ihre Studien 1927 mit dem Doktorgrad der Medizin („MD“) abzuschließen. Bereits 2 Jahre zuvor hatte sie, ihre vorausge-

\author{
H. E. Ulmer \\ Heidelberg, Deutschland
}

\title{
Helen B. Taussig
}

gangenen kardiologischen Erfahrungen nutzend, ihre erste wissenschaftliche $\mathrm{Pu}$ blikation über „Muskelkontraktionen im Büffelherzen“ veröffentlicht.

Ihr Mentor für die kommenden Jahrzehnte, der Pädiater Prof. Edwards A. Park, stellte sie vor die Aufgabe, sich des bis dahin mangels therapeutischer Optionen vernachlässigten $\mathrm{Be}$ reichs der angeborenen und erworbenen Herzfehler bei Kindern anzunehmen. $\mathrm{Zu}$ diesem Zeitpunkt, am Beginn einer möglichen kardiologischen Karriere, traf sie ein weiterer harter Schicksalsschlag. Sie entwickelte, wohl als Folge einer in der Kindheit durchgemachten schweren Keuchhustenerkrankung, eine zunehmende Schwerhörigkeit, die zeitweise bis zur Taubheit führte, und ihr dadurch die Möglichkeit der Nutzung der Auskultation zur kardiologischen Diagnostik so gut wie unmöglich machte. Sie lernte Lippenlesen und die Auskultation durch feinfühlige Palpation mit den Fingern soweit zu ersetzen, wie es ging. Dadurch standen auch, zumindest über einen längeren Zeitraum, neben der sorgfältigen Anamnese und Inspektion ausführliche und spezielle Röntgenuntersuchungen des Herzens im Vordergrund ihrer kardiologischen Diagnostik. Die Nachfrage vieler Patienten und deren Eltern nach einer spezialisierten Betreuung war rasch so groß, dass Dr. Park bereits 1930 die erste große kinderkardiologische Spezialambulanz, das später so bekannte und berühmte Harriett Lane Home, in Baltimore einrichtete und deren Leitung an Helen Taussig übertrug. Diese Position sollte sie im weiteren Verlauf mehr als 30 Jahre ununterbrochen und mit großem Erfolg einnehmen.

Mit dem ersten operativen Verschluss eines persistierenden Ductus arteriosus Botalli durch den Chirurgen Robert
Gross im August 1938 im Children's Hospital Boston eröffneten sich für den Bereich der angeborenen Herzfehler grundlegend neue Perspektiven. Diese Entwicklung wurde auch in der Johns Hopkins University aufgegriffen und nachhaltig verfolgt. Von größter Bedeutung für die damalige Zeit war die in Baltimore entwickelte sog. BlalockTaussig-Anastomose, eine Operation zur Verbesserung der Lungendurchblutung bei zyanotischen Vitien wie z.B. der Fallot-Tetralogie. Diese Operation trat nach ihrer ersten erfolgreichen Durchführung im November 1944 von Baltimore aus einen unvergleichlichen Siegeszug um die ganze Welt an und half, viele Leben zu retten. Die Geschichte der Operationsmethode wird im Beitrag „Alfred Blalock“ der „Historischen Profile“ näher geschildert.

Zur Zeit ihrer ersten umfassenden Darstellung angeborener Herzfehler, "Congenital malformations of the heart"

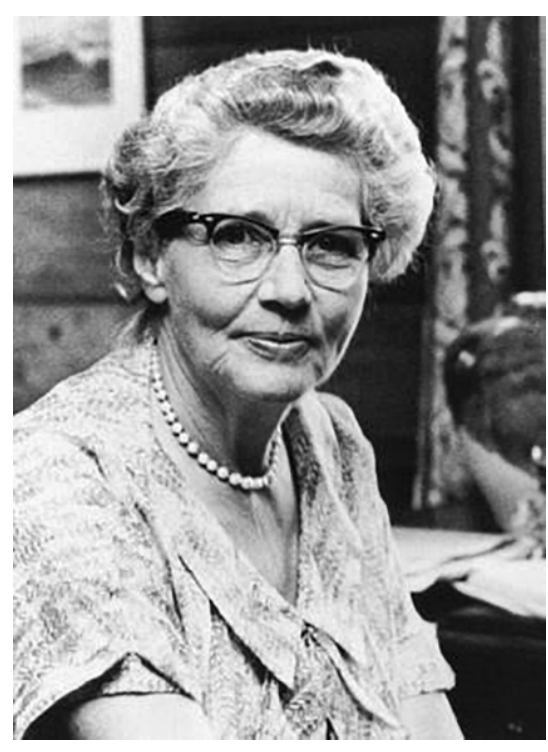

Abb. 1 ॥ Helen B. Taussig (1898-1986). (Aus [1]) 


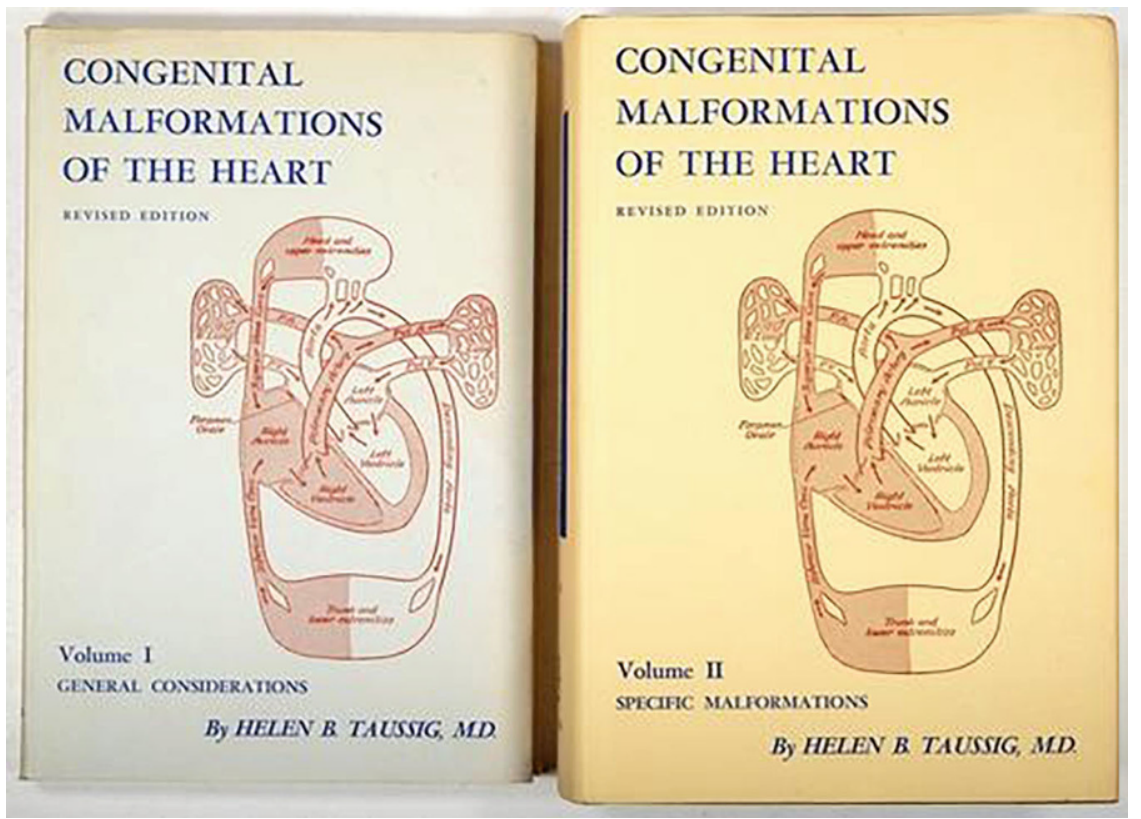

Abb. 2 ム Cover der Erstauflage des ersten klinischen Lehrbuchs über angeborene Herzfehler von Helen Taussig. (The Commonwealth Foundation, New York, 1947)

(• Abb. 2), mit deren Abfassung Helen Taussig bereits 1937 begonnen hatte, waren Operationen angeborener Fehlbildungen des Herzens noch ein Traum gewesen, und diesbezügliche Erfahrungen waren entsprechend hierin noch nicht enthalten. Diese mussten nun in das umfangreiche zweibändige Werk bis zum Erscheinen seiner Erstauflage im Jahre 1947 noch nachträglich eingearbeitet werden. Über mehrere Jahrzehnte und mehrere Generationen nachfolgender Kinderkardiologen hinweg galt diese einzigartige Monographie dann als die „Bibel“ der Kinderkardiologie.

Obwohl bis 3 Jahre vor ihrem Ausscheiden aus dem aktiven Dienst lediglich als Associate Professor eingestuft und erst 12 Jahre später als ihr chirurgischer Kollege Alfred Blalock, zum (ersten weiblichen) Full Professor ihrer Universität ernannt, wuchsen ihre Bekanntheit und ihr Einfluss auf diesem neuen Gebiet sehr rasch. So war sie eine der 6 Gründerinnen des ersten „Sub-Boards“ für Pädiatrische Kardiologie. Zahlreiche Vorträge und Invited Lectures auf der ganzen Welt, bei denen sie noch immer mit Problemen ihrer Dyslexie zu kämpfen hatte, machten sie und ihre Wirkungsstätte weltbekannt. Erst 3 Jahre vor ihrer Emeritierung im Sommer 1963 ermöglichte ihr die Operation ihrer Otosklerose erstmals die Auskultation des kindlichen Herzens mit einem normalen Stethoskop.

Allerdings wollte und konnte sich $\mathrm{He}$ len Taussig auch nach ihrem offiziellen Ausscheiden nicht aus dem akademischen Leben zurückziehen. Im Jahr 1965 wurde sie als erste Frau zur Präsidentin der American Heart Association gewählt. Auch 42 ihrer 100 wichtigsten Publikationen sind erst nach 1963 entstanden, wovon die letzte noch 1988, d.h. 2 Jahre nach ihrem Tod, erschienen ist.

Ihr wichtigster Beitrag aus dieser Zeit betraf die Thalidomid-Affäre. Von ihrem ehemaligen Fellow Dr. Alois Beuren, inzwischen Professor und erster kinderkardiologischer Lehrstuhlinhaber in Deutschland, wurde sie auf einen möglichen Zusammenhang zwischen der Verwendung dieses Pharmakons bei Schwangeren und dem Auftreten von Phokomelien und angeborenen Herzfehlern aufmerksam gemacht. Nach Überprüfung dieses Verdachts konnte sie durch ihren vehementen Einsatz vor dem amerikanischen Kongress die Zulassung dieser Substanz in den Vereinigten Staaten verhindern. Dafür wurde sie 1964 vom amerikanischen Präsidenten mit der Freiheitsmedaille der Vereinigten Staaten ausgezeichnet.

Am 20. Mai 1986, d. h. 3 Tage vor ihrem 88. Geburtstag, verstarb Helen Taussig bei einem von ihr unverschuldeten Verkehrsunfall mit ihrem Auto auf dem Weg $\mathrm{zu}$ einer Lokalwahl.

\section{Korrespondenzadresse}

\section{Prof. Dr. H. E. Ulmer}

Am Aukopf 5, 69118 Heidelberg, Deutschland herbert_ulmer@t-online.de

\section{Literatur}

1. U.S. National Library of Medicine, Digital Collections. 8600 Rockville Pike, Bethesda, MD 20894 